\title{
A Rule-Based Approach to Implicit Emotion Detection in Text
}

\author{
Orizu Udochukwu and Yulan He \\ School of Engineering and Applied Science \\ Aston University, UK \\ \{orizuus, y.he9\}@aston.ac.uk
}

\begin{abstract}
Most research in the area of emotion detection in written text focused on detecting explicit expressions of emotions in text. In this paper, we present a rule-based pipeline approach for detecting implicit emotions in written text without emotion-bearing words based on the OCC Model. We have evaluated our approach on three different datasets with five emotion categories. Our results show that the proposed approach outperforms the lexicon matching method consistently across all the three datasets by a large margin of $17-30 \%$ in $\mathrm{F}$-measure and gives competitive performance compared to a supervised classifier. In particular, when dealing with formal text which follows grammatical rules strictly, our approach gives an average F-measure of $82.7 \%$ on "Happy", "Angry-Disgust" and "Sad", even outperforming the supervised baseline by nearly $17 \%$ in F-measure. Our preliminary results show the feasibility of the approach for the task of implicit emotion detection in written text.
\end{abstract}

Keywords: Implicit emotions, OCC model, emotion detection, rulebased approach

\section{Introduction}

Human emotions are defined as subjective feelings and thoughts, and is a short episode that is coordinated by the brain [4]. Emotions exist in various forms and Ekman [2] made a strong compelling case for the six basic emotion categories. In Natural language Processing (NLP), emotion detection focuses on categorising a piece of text into an emotion category. The expression of emotion in written text is through the use of words and most often emotion-bearing words such as "happy". However, emotions can be adequately expressed without the use of emotion-bearing words. For example, given two sentences "The outcome of my exam makes me happy." and "I passed my exam." , both sentences express the emotion of happiness, with the first expressing it explicitly and the second implying it. Most research in the area of emotion detection focuses on explicit emotion detection $[9,6]$. Implicit emotion detection is a much more difficult task and the approaches which rely on emotion lexicons are inapplicable here. Although it is possible to train supervised classifiers from annotated data, acquiring sufficient annotated data for training requires heavy manual effort. 
We present in this paper a rule-based approach to sentence-level implicit emotion detection based on the OCC model, which was created by Ortony, Clore \& Collins in their book "The Cognitive Structure of Emotions" [7] and over the years has become a widely accepted model for emotion. we demonstrate using this resource and with the use of NLP techniques we are able to detect and classify implicit expressions of emotion in text. As opposed to most existing approaches, our approach does not rely on any specific knowledge base or annotated training data and can still offer a reasonably high precision rate given the complex nature of the problem.

\section{The OCC Model}

The OCC Model provides a clear and convincing structure of the eliciting conditions of emotions and the variables that affect their intensities [7]. It describes a hierarchy that classifies 22 emotion types. The hierarchy contains three branches. The first branch is the "consequences of events" branch. The emotions on this branch express pleasure or displeasure with event consequences. The second branch contains emotions in relation to "actions of agents". An agent can be self or other and these are the attribution type of emotions. The third branch contains emotions relating to liking or disliking in regards of "aspects of objects".

The OCC model is dependent on its variables and the rules for implementing/identifying emotion. The variables are grouped into emotion-inducing variable and emotion intensity variables. For a full list of the emotions and variables, see [7]. The OCC model however is rather complex and full of ambiguity. Steunebrink et al. [10] outlined a number of issues of the original OCC model and proposed changes to remove duplications and ambiguities. In our work, we use the revisited OCC model [10] for emotion detection in text.

\section{Our Approach}

In order to use the OCC model for emotion detection, we need to first assign values to a list of variables defined in OCC and then use a set of pre-defined rules to identify an emotion for a given text. In this paper, we focus on identifying emotion in relation to events and actions only and leave the detection of emotions associated with objects as future work. The list of rules is shown in Table 1. For example, the first row of Table 1 (a) can be read as

If Direction = "Self" and Tense = "Future" and Overall Polarity = "Positive" and Event Polarity = "Positive", then Emotion = "Hope".

It is worth noting that emotion-bearing words are different from polaritybearing words. An emotion-bearing word can be described as words which on their own can convey emotions. For example, the word "passionate" can convey an emotion of Joy. Polarity-bearing words, on the contrary, express positive or negative polarity in a given context. For example, the word "pass" expresses a positive polarity as in "I passed my exam.". But the word "pass" does not have an explicit prior emotion associated with it. Hence, it is more likely that 
emotions-bearing words also have a polarity, but not all polarity words convey specific emotions.

Table 1. Rules for emotion detection.

\begin{tabular}{llll|l}
\hline Direction & Tense & $\begin{array}{l}\text { Input Variables } \\
\text { Overall polarity }\end{array}$ & Event polarity & $\begin{array}{l}\text { Output } \\
\text { Emotion }\end{array}$ \\
\hline Self & Future & Positive & Positive & Hope \\
Self & Future & Negative & Negative & Fear \\
Self & Present & Positive & Positive & Joy \\
Self & Present & Negative & Negative & Distress \\
Self & Past & Positive & Positive & Satisfaction \\
Self & Past & Negative & Negative & Fears-confirmed \\
Self & Past & Positive & Negative & Relief \\
Self & Past & Negative & Positive & Disappointment \\
Other & All & Positive & Positive & Happy-for \\
Other & All & Negative & Positive & Resentment \\
Other & All & Positive & Negative & Gloating \\
Other & All & Negative & Negative & Sorry-for \\
\hline
\end{tabular}

(a) Event-based.

\begin{tabular}{|c|c|c|}
\hline \multicolumn{2}{|c|}{ Input Variables } & Output \\
\hline Direction & Polarity & Emotion \\
\hline Self & Positive & Pride \\
\hline Self & & \\
\hline Other & Pos & niration \\
\hline Other & Negative & Reproach \\
\hline
\end{tabular}

(b) Action-based.

\begin{tabular}{|c|c|c|}
\hline Input & Variables & Output \\
\hline Event & Action & Emotion \\
\hline Joy & $\begin{array}{l}\text { Pride } \\
\text { Shame }\end{array}$ & Gratification \\
\hline Distress & $\begin{array}{l}\text { Shame } \\
\text { Admiration }\end{array}$ & $\begin{array}{l}\text { Remorse } \\
\text { Gratitude }\end{array}$ \\
\hline Distress & Reproach & Anger \\
\hline
\end{tabular}

(c) Compound emotions.

We first perform pre-processing on text in order to be able to assign values to the OCC variables which will be discussed subsequently. For pre-processing we carried out sentence splitting and tokenisation, part-of-speech (POS) tagging, word sense disambiguation (WSD), dependency parsing, sentence tense detection based on the POS tags, and polarity detection using majority vote based on the lexicon matching results obtained with three sentiment lexicons, SentiWordNet [3], AFINN [5] and the Subjectivity Lexicon[12].

We now describe how we assign values to each OCC variable.

Direction: The value for this variable can either be "Self" or "Other". The former refers to emotions expressed for oneself while the latter refers to emotions expressed for others. This value is assigned based on the dependency relationship (identified by the dependency parser) of a first person pronoun (such as "I" , "we") with an action or event. We identify 3 possible scenarios for assigning a value to this variable: 1 ) When dealing with a simple sentence, we simply apply the process mentioned above; 2) When dealing with a complex sentence where multiple subject(s) are identified by the parser, we assign values based on respective action/event relations with identified subjects; 3) No subject is identified or no verbs exist in the text, here we just assign the value "Other" to the variable.

Tense: The value for this variable can either be "Present", "Past" or "Future". The value assignment is determined by the POS tags of the verbs in a sentence 
or by the results obtained from the FrameNet ${ }^{1}$ where the tense of the verb is identified as future tense if the frame is associated with "desiring". In the cases where no verbs are used in a sentence, the value of the variable is set to "Present".

Overall Sentence Polarity: The value for this variable can either be "Neutral", "Negative" or "Positive". It is determined by polarity detection through majority vote.

Event Polarity: The event is identified based on the verb-object relations revealed by the dependency parser. The noun phrase which contains an identified object is treated as the event for its relative verb. The polarity of an event is then determined using lexicon-matching.

Action Polarity: The action is identified based on the subject-verb relations revealed by the dependency parser. The verb phrase which contains the identified verb is treated as an action. Similar to event polarity, the action polarity is also determined using lexicon-matching.

We have also implemented a contextual valence shifter as described in [8] to detect polarity change in different context. Once the variable values are identified, the rules defined in Table 1 are then applied to detect the presence of emotions. The compound emotions are results of the output of the event-based and action-based emotions. For the "sorry-for" emotion, we ensure that the subject is of positive valence; otherwise the emotion is identified as "resentment". The same rule is applied to the "admiration" and "reproach" emotion pairs.

\section{Experiments}

In this section, we present the evaluation results of our rule-based emotion detection approach on three different datasets, which include:

- The International Survey On Emotion Antecedents And Reactions (ISEAR) Dataset $^{2}$ which was developed by asking nearly 3,000 participants from different cultural background about their emotional experiences.

- The SemEval-2007 Task 14 Affective Text dataset [11] consists of news headlines collected from major newspapers.

- The Alm's Dataset [1] comprises sentences taken from 176 fairy tale stories. We use only the data extracted from Grimm's and Anderson's tales, which have a total number of 1,040 sentences.

As our goal is to detect emotions in the absence of emotion-bearing words, we filter out sentences which contain emotion words as can be found in the emotion lexicon WordNet-Affect ${ }^{3}$. The total number of sentences before and after filtering of emotion-bearing words in each emotion category for these three datasets are shown in Table 2. We focus specifically on the 5 emotion categories which are

\footnotetext{
1 https://framenet.icsi.berkeley.edu/fndrupal/

${ }^{2}$ http://www.unige.ch/fapse/emotion/databanks/isear.html

3 http://wndomains.fbk.eu/wnaffect.html
} 
shared across these three datasets and map the OCC-output emotions to the five emotion categories in the following ways: (Fear, Fear-confirmed) $\rightarrow$ Fear, (Joy, Happy-For, Satisfaction, Admiration, Pride) $\rightarrow$ Joy, (Anger, Reproach) $\rightarrow$ Anger, (Distress, Sorry-For, Disappointment, Shame) $\rightarrow$ Sadness, Resentment $\rightarrow$ Disgust.

Table 2. Statistics of the datasets. "Total" denotes the original number of sentences in each emotion category while "Implicit" denote the number of sentence which do no contain any emotion words according to WordNet-Affect.

\begin{tabular}{lcc}
\hline Emotion & Total Implicit \\
\hline Joy & 1095 & 537 \\
Fear & 1095 & 366 \\
Anger & 1096 & 483 \\
Sadness & 1096 & 488 \\
Disgust & 1096 & 484 \\
Shame & 1096 & 581 \\
Guilt & 1093 & 482 \\
\hline Total & 7667 & 3421 \\
\hline
\end{tabular}

(a) ISEAR

\begin{tabular}{lcc}
\hline Emotion & Total & Implicit \\
\hline Joy & 362 & 317 \\
Fear & 160 & 130 \\
Anger & 66 & 60 \\
Sadness & 202 & 182 \\
Disgust & 26 & 24 \\
Surprise & 184 & 160 \\
\hline Total & 1000 & 873 \\
\hline
\end{tabular}

(b) SemEval

\begin{tabular}{|c|c|c|}
\hline Emotion & Total & mplicit \\
\hline Нарpy & 406 & 103 \\
\hline Fearful & 121 & 33 \\
\hline Angry-Disgusted & 174 & 84 \\
\hline Sad & 247 & 90 \\
\hline Surprised & 92 & 50 \\
\hline Total & 1040 & 360 \\
\hline
\end{tabular}

(c) Alm's

We have developed two baseline models. One is a lexicon matching method which uses the NRC emotion Lexicon ${ }^{4}$ for sentence-level emotion detection, We also train supervised Naïve Bayes (NB) classifiers using the implementation in Weka $^{5}$ on the three datasets with 5 -fold cross validation.We report the results in terms of the F-measure scores.

Table 3. Performance comparison of F-measure results on the three datasets. Bold face values denote the best results obtained in each dataset.

\begin{tabular}{l|ccc|ccc|ccc}
\hline \multirow{2}{*}{ Emotion } & \multicolumn{3}{|c|}{ ISEAR } & \multicolumn{3}{c|}{ SemEval } & \multicolumn{3}{c}{ Alm's } \\
\cline { 2 - 10 } & Lexicon & NB & Rule & Lexicon & NB & Rule & Lexicon & NB & Rule \\
\hline Joy/Happy & 33.4 & 61.2 & $\mathbf{6 9 . 6}$ & 39.7 & $\mathbf{7 1 . 7}$ & 59.9 & 58.8 & 63.5 & $\mathbf{8 1 . 8}$ \\
Fear/Fearful & 0 & $\mathbf{4 7 . 6}$ & 18.3 & 0 & $\mathbf{5 2 . 2}$ & 31.8 & 0 & $\mathbf{2 6 . 7}$ & 14.0 \\
Anger/Angry-Disgusted & 23.0 & 47.1 & $\mathbf{6 1 . 3}$ & 55.8 & 16.2 & $\mathbf{6 1 . 3}$ & 48.9 & 58.6 & $\mathbf{8 6 . 6}$ \\
Sadness/Sad & 25.6 & 55.4 & $\mathbf{6 8 . 0}$ & 47.8 & 56.0 & $\mathbf{7 1 . 5}$ & 61.0 & 56.0 & $\mathbf{7 9 . 6}$ \\
Disgust & 25.6 & $\mathbf{5 1 . 0}$ & 39.2 & 38.5 & 34.5 & $\mathbf{6 1 . 7}$ & - & - & - \\
\hline Average & 21.5 & $\mathbf{5 2 . 5}$ & 51.3 & 36.4 & $\mathbf{5 8 . 2}$ & 57.3 & 42.2 & 56.0 & $\mathbf{6 5 . 5}$ \\
Average (- Fear) & 27.0 & 53.7 & $\mathbf{5 9 . 5}$ & 45.5 & 44.6 & $\mathbf{6 3 . 6}$ & 56.12 & 65.8 & $\mathbf{8 2 . 7}$ \\
\hline
\end{tabular}

It can be observed from Table 3 that although we have filtered out sentences which contain emotion words from WordNet-Affect, using other emotion lexicons

\footnotetext{
${ }^{4}$ http://www. saifmohammad.com/WebPages/lexicons.html

${ }^{5}$ http://www.cs.waikato.ac.nz/ml/weka
} 
such as the NRC emotion lexicon can still identify emotions of some sentences. Nevertheless, using the NRC lexicon gives quite low F-measure values across the three datasets and it fails to detect any "Fear" emotion bearing sentences. Despite using no labelled data, our approach achieves similar performance as supervised NB on the ISEAR and SemEval datasets (with $1 \%$ difference in Fmeasure on average) and outperforms NB by $9.5 \%$ in F-measure on the Alm's dataset. The results also show that our approach is largely affected by the quality of text. ISEAR contains personal experience expressed by a wide range of participants and hence might contain lots of informal and ill-grammatical text. SemEval contains news headlines which are often incomplete sentences ignoring grammar conventions. The Alm's dataset, on the other hand, contains fairy tales which are formal text following rules of grammar very strictly. As such, the performance obtained from the Alm's dataset by our approach are significant better than that obtained from the other two datasets.

Our approach relies on results generated from a series of NLP tasks such as POS tagging,word-sense disambiguation, dependency parsing and polarity detection in order to be able to assign values to a set of OCC variables for emotion detection. Thus, any error occurred will be propagated down the pipeline process. Furthermore, failure in detecting the polarity of text will make it impossible for our approach to identify the underlying emotion. Also, we have not considered ironic and sarcastic sentences in our current work. Nevertheless, we have shown that in the absence of annotated data, the OCC-based approach is able to identify implicit emotion in text with performance competing to supervised classifiers and it even outperforms the supervised approach for formal text (the Alm's dataset). The emotion detection results generated by the OCC-based approach can be used as seed examples to bootstrap more complicated emotion detection methods which require large amount of training data. We will leave it as our future work.

\section{Conclusions and Future Work}

In this paper, we have proposed a OCC-based approach for implicit emotion detection. Experimental results on three datasets have shown that our approach outperforms the lexicon matching method consistently across all the three datasets by a large margin of 17-30\% in F-measure and gives competitive performance compared to a supervised method. Also, when dealing with formal text which follows grammatical rules strictly, the approach achieves an average F-measure score of $82.7 \%$ in identifying "Happy", "Angry-Disgusted" and "Sad" categories.

In future, we will investigate methods to improve the performance of our approach with informal short text such as tweets and social media posts. We will also improve the identification of emotions involving intensity variables and unexpectedness variables by examining how adverbs and adjectives influence the emotion of sentences (for emotions like "Surprise" and "Shock") and investigate performance on ironic or sarcastic sentences. We will study possible solutions to deal with the poor performing emotion category such as "Fear". 


\section{References}

1. Alm, C.O., Roth, D., Sproat, R.: Emotions from text: machine learning for textbased emotion prediction. In: Proceedings of the conference on Human Language Technology and Empirical Methods in Natural Language Processing. pp. 579-586 (2005)

2. Ekman, P.: An argument for basic emotions. Cognition \& Emotion 6(3-4), 169-200 (1992)

3. Esuli, A., Sebastiani, F.: Determining the semantic orientation of terms through gloss classification. In: Proceedings of the 14th ACM international conference on Information and knowledge management. pp. 617-624 (2005)

4. Friedenberg, J., Silverman, G.: Cognitive science: an introduction to the study of mind. Sage (2011)

5. Hansen, L.K., Arvidsson, A., Nielsen, F.Å., Colleoni, E., Etter, M.: Good friends, bad news-affect and virality in twitter. In: Future information technology, pp. 3443. Springer (2011)

6. Marsella, S., Gratch, J., Petta, P.: Computational models of emotion. A Blueprint for Affective Computing-A sourcebook and manual pp. 21-46 (2010)

7. Ortony, A.: The cognitive structure of emotions. Cambridge university press (1990)

8. Polanyi, L., Zaenen, A.: Contextual valence shifters. In: Computing attitude and affect in text: Theory and applications, pp. 1-10. Springer (2006)

9. Shivhare, S.N., Khethawat, S.: Emotion detection from text. arXiv preprint arXiv:1205.4944 (2012)

10. Steunebrink, B.R., Dastani, M., Meyer, J.J.C.: The occ model revisited. In: Proceedings of the 4th Workshop on Emotion and Computing. vol. 65, pp. 2047-2056 (2009)

11. Strapparava, C., Mihalcea, R.: Semeval-2007 task 14: Affective text. In: Proceedings of the 4th International Workshop on Semantic Evaluations. pp. 70-74. SemEval '07 (2007)

12. Wilson, T., Wiebe, J., Hoffmann, P.: Recognizing contextual polarity in phraselevel sentiment analysis. In: Proceedings of the conference on human language technology and empirical methods in natural language processing. pp. 347-354. Association for Computational Linguistics (2005) 